\title{
MicroRNA differential expression spectrum and microRNA-125a-5p inhibition of laryngeal cancer cell proliferation
}

\author{
XIANG-DONG YAO, PING LI and JI-SHENG WANG \\ Ear, Nose and Throat Department, Xinxiang Central Hospital, Xinxiang, Henan 453000, P.R. China
}

Received December 9, 2015; Accepted January 6, 2017

DOI: $10.3892 / \mathrm{etm} .2017 .4685$

\begin{abstract}
The present study aimed to screen and analyze the differential expression spectrum of microRNA (miRNA) between laryngeal cancer tissue and surrounding normal laryngeal mucosa in order to provide an indication for further study to determine the role of miRNA in the initiation and development of laryngeal cancer. A total of 42 pairs of specimens of laryngeal carcinoma tissues and adjacent normal laryngeal mucosa were collected. A total of 10 pairs of specimens were randomly selected for miRNA microarray gene chip analysis, and the remaining 32 pairs of specimens were used for reverse transcription-quantitative polymerase chain reaction (RT-qPCR) verification to identify miRNA that were differentially expressed in laryngeal cancer tissues. Methylthiazolyldiphenyl-tetrazolium bromide and clone formation assays were utilized to elucidate the physiological relevance of the miRNA miR-125a-5p on the proliferation of laryngeal cancer human epithelial type 2 (Hep2) cells. Results demonstrated that the expression levels of six miRNA were significantly downregulated in laryngeal carcinoma tissue, as identified by gene chip analysis and RT-qPCR $(\mathrm{P}<0.05)$. The six miRNA included let-7f-5p, miR-10a-5p, miR-125a-5p, miR-144-3p, miR-195-5p and miR-203. Compared with the control group, the proliferative ability of laryngeal cancer Hep2 cells was inhibited in a transfected miR-125a-mimics group. In contrast, proliferation was promoted in a transfected miR-125a-inhibitor group In conclusion, the results of gene chip analysis were consistent with that of RT-qPCR. Results demonstrated that miRNA in laryngeal cancer and normal laryngeal mucosa exhibited evident differential expression, which may contribute to the laryngeal cancer incidence and invasion. miR-125a was able to inhibit the proliferation of
\end{abstract}

Correspondence to: Dr Xiang-Dong Yao, Ear, Nose and Throat Department, Xinxiang Central Hospital, 56 Jinsui Road, Xinxiang, Henan 453000, P.R. China

E-mail: xinxiangzhoujie@163.com

Key words: laryngeal, cancer, microRNA, microarray chip, cell proliferation
Hep2 laryngeal cancer cells and, therefore, may serve as a novel target for laryngeal cancer biological therapy.

\section{Introduction}

Head and neck neoplasm represents a major type of malignancy that adversely affects human life. Its incidence rate is ranked sixth among all cancer types (1). Laryngeal carcinoma is one of the most common head and neck tumors, and its incidence rate is ranked second in respiratory tract cancer types, the majority of which are considered as squamous-cell carcinoma (1). Surveys from around the world have demonstrated that the incidence of laryngeal cancer is increasing by $\sim 25 \%$ per year $(2,3)$. The worldwide incidence rate of laryngeal cancer in males is $\sim 5.1 / 100,000$ and the mortality rate of male patients was $\sim 2.2 / 100,000$ in 2008 (1). Previous studies have demonstrated that, despite surgical advances over the last 30 years with application of chemotherapeutic drugs and more advanced radiation therapy methods in the treatment of laryngeal cancer, as in other head and neck cancers, the overall survival rate of patients with laryngeal cancer has declined $(4,5)$. Epidemiological investigation exhibited that possible causes of laryngeal cancer include smoking, alcohol, air pollution and occupational factors (6,7). Although various studies have indicated that the occurrence and development of laryngeal cancer is correlated with tumor-promoting cancer genes [including B-cell lymphoma 2 (Bcl-2) and c-Myc] and tumor-suppressive cancer genes (including p53, Rb, P16 and p21) $(8,9)$, the molecular mechanisms of laryngeal cancer remain elusive, thus presenting a challenge in intervening in the pathogenesis of laryngeal cancer and improving its survival rate.

microRNA (miRNA) are a small class of 19-25 nt non-coding RNA. miRNA control the biology of the 3' untranslated region of mRNA, which can degrade, inhibit the translation of and regulate the expression level of target genes, and subsequently participate in the regulation of cell apoptosis, proliferation and differentiation $(10,11)$. Previous studies suggest that miRNA may have an important role in the initiation, development, invasion, translation and angiogenesis of cancer and tumor suppressor genes $(12,13)$ and may be associated with the occurrence and development of head and neck cancer (14). This suggests that miRNA may have potential clinical applications in the early diagnosis, treatment 
and prognosis of laryngeal carcinoma as a biomarker or therapeutic target.

The differential expression of miRNA in laryngeal carcinoma has not been fully elucidated. In the present study, the next-generation miRCURY LNA microarray chip of all human miRbase in the miRNA database was utilized to screen the differential expression of miRNA in laryngeal carcinoma and was verified by reverse transcription-quantitative polymerase chain reaction (RT-qPCR) analysis. Furthermore, the effect of the miRNA miR-125a-5p on the proliferation of human epithelial type 2 (Hep2) cells was investigated. The aim of the present study was to provide an indication for further investigation of the relationship between miRNA and the occurrence and development of laryngeal carcinoma, in order to improve understanding of the pathogenesis of laryngeal cancer.

\section{Materials and methods}

Ethics statement. For experiments involving human subjects, approval was obtained from the institutional review board of Xinxiang Central Hospital (Xinxiang, China). Informed consent was provided according to the Declaration of Helsinki and written informed consent was provided by each patient providing biopsy samples for the experiments.

Collection of tissue samples and clinical data. A total of 42 pairs of laryngeal carcinoma tissues and adjacent normal tissues were collected between October 2007 and March 2009 in Xinxiang Central Hospital (Henan, China). During surgery, samples $\left(\sim 0.5 \mathrm{~cm}^{3}\right)$ of normal laryngeal mucosa were cut from the laryngeal carcinoma tissues with a negative surgical margin of at least $2 \mathrm{~cm}$. Samples were rinsed with saline and subsequently stored in RNAlater RNA stabilization reagent (Qiagen China Co., Ltd., Shanghai, China) at $-80^{\circ} \mathrm{C}$. Clinical pathological data of 42 patients with laryngeal carcinoma are presented in Table I. Patients had no previous history of radiotherapy and chemotherapy treatment. Patients were randomly divided into two groups: 10 patient samples were tested using miRNA microarray analysis and the remaining 32 samples were analyzed using RT-qPCR.

Total RNA extraction and verification. TRIzol reagent (Invitrogen; Thermo Fisher Scientific, Inc., Waltham, MA, USA) and miRNeasy mini kit (Qiagen China Co.) were used to extract the total RNA and miRNA from the laryngeal samples, according to the manufacturer's instructions. Nanodrop apparatus (ND-1000; NanoDrop Technologies; Thermo Fisher Scientific, Inc.) was used to confirm successful RNA extraction and to determine the quality of the RNA. The integrity of RNA was determined by gel electrophoresis (data not shown).

miRNA microarray determination and analysis

Fluorescent labeling and chip hybridization for RNA. A total of 10 specimens were selected randomly for analysis using an miRNA microarray. Following RNA extraction, miRNA were labelled using the miRCURY Hy3/Hy5 Power labeling kit (Exiqon Inc., Woburn, MA, USA), according to the manufacturer's instructions. Subsequent to fluorescent labeling, the specimens labelled with Hy3 were hybridized to the chip and the miRCURY LNA Array v.16.0 software (Exiqon, Inc.) was utilized to study miRNA expression, according to the manufacturer's instructions.

Cleaning, scanning and signal processing. Following miRNA hybridization with the miRNA chip array, the chip was washed several times with the elution buffer (Exiqon, Inc.) and centrifuged for $5 \mathrm{~min}$ at $4^{\circ} \mathrm{C}(61 \mathrm{x} \mathrm{g})$ to dry. The chip was subsequently scanned with the Axon GenePix 4000B Microarray Scanner (Axon; Molecular Devices, LLC, Sunnyvale, CA, USA).

The scanned image was input into Pro GenePix 6 (Axon; Molecular Devices, LLC) software for coordinate adjustment and data extraction. The mean miRNA data were obtained from multiple duplicates, and normalized factors were calculated for miRNA with an intensity of $\geq 50$ in all samples. Expression data were normalized using the median. Significantly differentially expressed miRNA were identified by Volcano Plot filtering. Cluster analysis was performed using MEV v.4.6 software (The Institute of Genomic Research Database, Rockville, MD, USA).

$R T-q P C R$ verification. A total of 32 pairs of laryngeal carcinoma tissues and adjacent normal mucosa tissue specimens were used for total RNA extraction. Following RNA extraction, the expression levels of the miRNA let-7f-5p, miR-10a-5p, miR-125a-5p, miR-144-3p, miR-195-5p and miR-203 in each sample was determined using RT-qPCR verification with stem-loop primers, using SYBR Green dye and U6 as the internal reference.

A total of $2 \mathrm{ng}$ to $2 \mu \mathrm{g}$ RNA from each total RNA extraction was used for RT-qPCR. For reverse transcription, the mixture was prepared as follows: All primers (Guangzhou RiboBio Co., Ltd., Guangzhou, China) were added to $24 \mu \mathrm{l}$ RNase free water, mixed, incubated for $10 \mathrm{~min}$ at $70^{\circ} \mathrm{C}$ and subsequently kept in an ice bath for $2 \mathrm{~min}$. Primer sequences were as follows: Forward, 5'-CTTGTCCTTCATTCCACC GCA-3' and reverse, 5'-TGCCGCCTGAACTTCACTCC-3'. Following this, $2 \mu \mathrm{l}$ RNase inhibitor, $8 \mu \mathrm{l}$ M-MLV buffer (5X), $2 \mu \mathrm{l}$ M-MLV reverse transcriptase (RNase $\mathrm{H}^{-}$) and $2 \mu \mathrm{l}$ dNTP (Takara Bio, Otsu, Japan) were added to the reaction mixture and RNase-free water was added to reach a total reaction volume of $40 \mu \mathrm{l}$. The reaction mixture was incubated for $10 \mathrm{~min}$ at $30^{\circ} \mathrm{C}, 1 \mathrm{~h}$ at $42^{\circ} \mathrm{C}$ and $15 \mathrm{~min}$ at $70^{\circ} \mathrm{C}$. The reverse transcripts obtained were used as PCR templates.

Amplification and fluorescence quantification were performed using a Bio-Rad thermocycler (Bio-Rad Laboratories, Inc. The total PCR mixture consisted of $10 \mu \mathrm{l}$ SYBR 2X Green I fluorescence reaction fluid (Beijing Bioteke Biological Technology Co., Ltd., Beijing, China), $0.25 \mu 1$ of each of the forward and reverse primers (10 $\mu \mathrm{mol} / \mathrm{l}$; forward, 5'-CCATACCACCCTGAACGC-3' and reverse, 5'-AGCCTA CAGCACCCGGTAT-3'), $1 \mu$ l sample template and RNase free water, to give a total reaction volume of $20 \mu l$. The PCR cycling conditions were as follows: Pre-denaturation step at $95^{\circ} \mathrm{C}$ for $20 \mathrm{sec}$, followed by $95^{\circ} \mathrm{C}$ for $10 \mathrm{sec}, 60^{\circ} \mathrm{C}$ for $20 \mathrm{sec}$ and $70^{\circ} \mathrm{C}$ for $10 \mathrm{sec}$, for 40 cycles. Subsequently, the $2^{-\Delta \Delta \mathrm{Cq}}$ method (15) was used to quantify the expression level of each miRNA relative to U6. 


\section{Cell cultivation}

Cell line, culture medium and related reagents and instruments. Laryngeal cancer Hep2 cells were purchased from ATCC (Manassas, VA, USA). RPMI-1640 basic culture medium and RPMI-1640 complete culture medium (Gibco; Thermo Fisher Scientific, Inc., Waltham, MA, USA) were utilized for cell culture. Fetal bovine serum (FBS; Gibco; Thermo Fisher Scientific, Inc.) and $0.25 \%$ trypsin (Gibco; Thermo Fisher Scientific, Inc.) were used as supplements and cells were cultured at $37^{\circ} \mathrm{C}$. Other culture reagents included $5 \mathrm{~g} / 1$ methylthiazolyldiphenyl-tetrazolium bromide (MTT; Sigma Aldrich; Merck Millipore, Darmstadt, Germany), dimethyl sulfoxide (DMSO; Sigma Aldrich; Merck Millipore) and hematoxylin. Equipment used for cell culture included 96- and 6-well plates (Corning Inc., Corning, NY, USA) and a decolorization shaker (Qilinberier Instrument Manufacturing Co., Ltd., Haimen China).

Transient transfection of Hep2 laryngeal carcinoma cells. Prior to transfection, Hep2 cells were digested and counted. Following this, $4 \times 10^{5}$ Hep2 cells were placed in each well of the 6-well plates. Subsequent to the cells reaching 70-80\% confluency, miR-125a-mimic, miR-125a-inhibitor (Bioneer Corp., Shanghai, China) or scrambled sequence oligonucleotide (negative control) were added to $250 \mu \mathrm{l}$ of the serum-free culture medium Opti-MEM (Gibco; Thermo Fisher Scientific, Inc.). Following this, $4 \mu \mathrm{l}$ FuGENE 6 Transfection Reagent (Promega Corp., Madison, WI, USA) was added to each well, the reaction was mixed and incubated at room temperature for $15 \mathrm{~min}$. Cells were washed twice with the basic culture medium (Opti-MEM) and $1.5 \mathrm{ml}$ basic serum-free culture medium (Opti-MEM) was subsequently added to the DNA-FuGENE 6 Transfection Reagent complex in the wells. The plates were swirled gently to mix and disperse the liquid evenly. Following $6 \mathrm{~h}$, the transfection complex was absorbed and discarded and fresh culture supplemented with $10 \%$ FBS was added.

MTT test. Hep2 laryngeal carcinoma cells were digested with trypsin and collected into a culture medium supplemented with FBS. Following centrifugation at $4^{\circ} \mathrm{C}$ for $2 \mathrm{~min}$ at $112 \mathrm{x} \mathrm{g}$, cells were counted and diluted to $0.5-1 \times 10^{7}$ cells $/ 1$. A total of $200 \mu \mathrm{l}$ of cell suspension was added to each well. According to cell type, cells were divided into four groups and cultured into four 96 -well plates in triplicates and maintained in an incubator at $37^{\circ} \mathrm{C}$. Cells were monitored at $1,3,5$ and 7 days following the addition of $20 \mu \mathrm{l}$ MTT $(5 \mathrm{~g} / \mathrm{l})$ to each well of each plate and, subsequently, the plates were incubated at $37^{\circ} \mathrm{C}$ for $4 \mathrm{~h}$. Following this, the MTT was discarded from the wells and $150 \mu \mathrm{l}$ DMSO was added to each well and mixed slowly using horizontal rotators. The absorbance (A) was recorded at $490 \mathrm{~nm}$ using a Bio-Rad microplate reader (Bio- $\mathrm{Rad}$ Laboratories, Hercules, CA, USA). The A values from days $1,3,5$ and 7 were collated and the growth curves of the cells were generated.

Clone formation experiment. Cells were collected at the logarithmic growth phase and the concentration of cell suspension was adjusted to $5 \times 10^{5}$ cells/well. These cells were divided into a 6-well plate with $2 \mathrm{ml}$ in each well (1,000 cells/well).
Table I. Clinicopathological characteristics of patients with laryngeal carcinoma $(n=42)$.

\begin{tabular}{|c|c|}
\hline Characteristic & Number $(\%)$ \\
\hline $\operatorname{Age}^{\mathrm{a}}$ & $59.8 \pm 11.7$ \\
\hline \multicolumn{2}{|l|}{ Sex } \\
\hline Male & $39(92.9)$ \\
\hline Female & $3(7.1)$ \\
\hline \multicolumn{2}{|l|}{ Cancer type } \\
\hline Supraglottic & $12(28.6)$ \\
\hline Glottic & $28(66.7)$ \\
\hline Subglottic & $2(4.8)$ \\
\hline \multicolumn{2}{|l|}{ Clinical stage } \\
\hline I & $19(45.2)$ \\
\hline II & $8(19.0)$ \\
\hline III & $2(4.8)$ \\
\hline IV & $13(31.0)$ \\
\hline \multicolumn{2}{|l|}{$\mathrm{T}$ stage } \\
\hline $\mathrm{T} 1$ & $19(45.2)$ \\
\hline & $10(23.8)$ \\
\hline $\mathrm{T} 3$ & $5(11.9)$ \\
\hline $\mathrm{T} 4$ & $8(19.1)$ \\
\hline No & $33(78.6)$ \\
\hline N1-N3 & $9(21.4)$ \\
\hline \multicolumn{2}{|l|}{ Differentiation } \\
\hline Poorly differentiated & $3(7.1)$ \\
\hline Moderately differentiated & $23(54.8)$ \\
\hline Highly differentiated & $16(38.1)$ \\
\hline
\end{tabular}

${ }^{\mathrm{a}}$ Mean \pm standard deviation. $\mathrm{T}$, tumor; $\mathrm{N}$, node.

Following 7-10 days, the growth of the clones was observed; a colony of $>50$ cells was considered to be one clone. Cells were washed three times with phosphate-buffered saline, pulsed and fixed with $1 \mathrm{ml} /$ well methanol and, subsequently, placed on a rotator for $10 \mathrm{~min}$. Following this, $1 \mathrm{ml} /$ well hematoxylin was added and the plates were placed on the rotator for a further $10 \mathrm{~min}$ before the hematoxylin was drained and discarded. The number of clones per well per plate was counted and images were captured. The clone formation rate was calculated as clone formation rate $(\%)=($ clone number $/$ incubation cell number) x 100 .

Statistical analysis. Statistical analysis was performed using SPSS v.13.0 (SPSS, Inc., Chicago, IL, USA). The experimental results of miRNA microarray were analyzed by the significance analysis of microarray (SAM) (Stanford University, Stanford, CA, USA), with a false discovery rate (FDR) of 0.05 to screen the differential expression of miRNA in laryngeal carcinoma tissues and adjacent tissues. Data were analyzed and a comparison between the two groups was performed using the independent samples $t$-test. Data are expressed as mean \pm standard deviation. $\mathrm{P}<0.05$ was considered to indicate a statistically significant difference. 


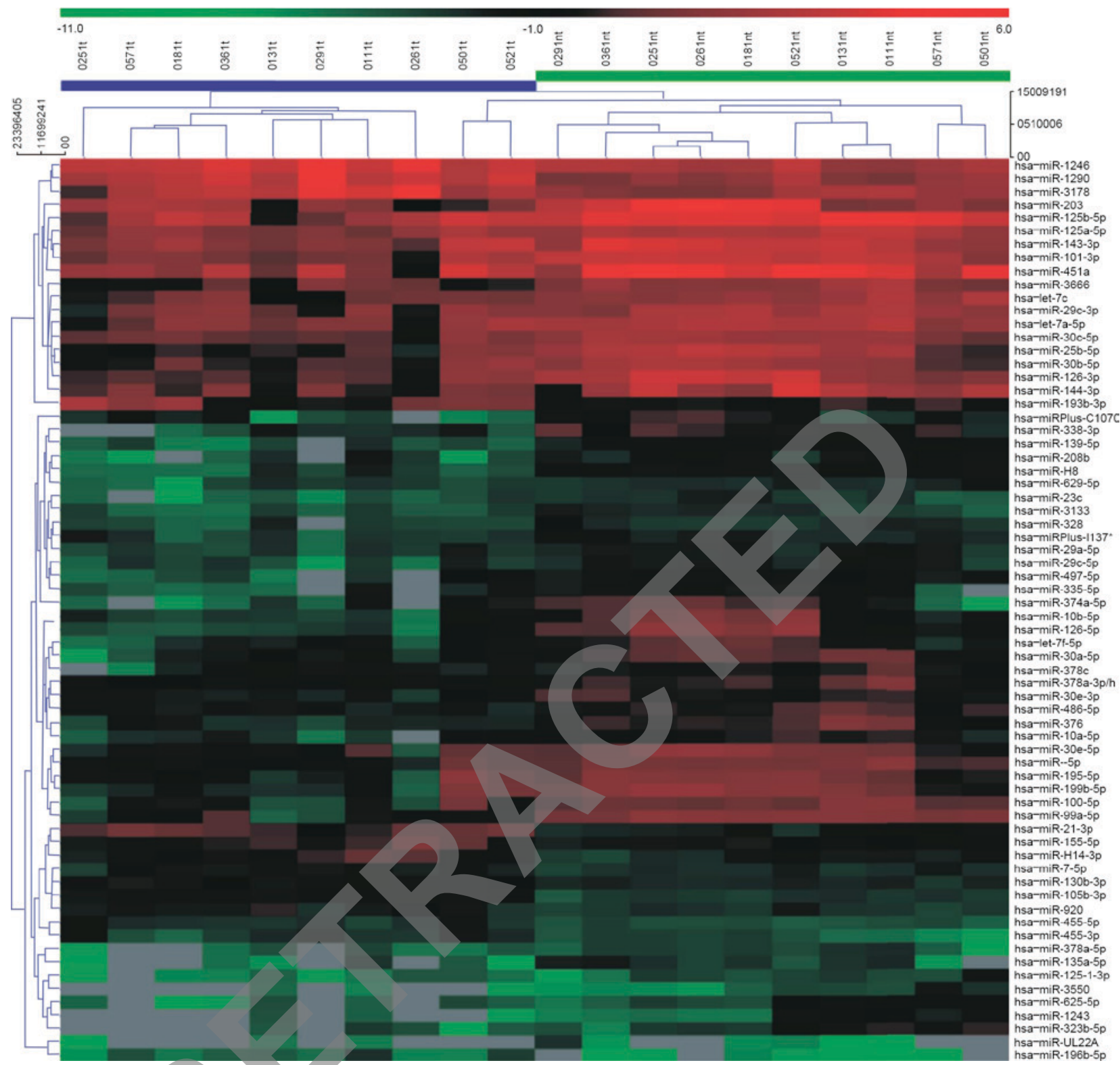

Figure 1. Heat map of miRNA microarray in laryngeal squamous cell carcinoma. Hierarchical clustering of differentially expressed miRNA are exhibited in paired tumor-normal samples. Tissue samples are represented in columns and differentially expressed miRNA are delineated in rows. Red indicates upregulation and green indicates downregulation of miRNA expression. miRNA, microRNA.

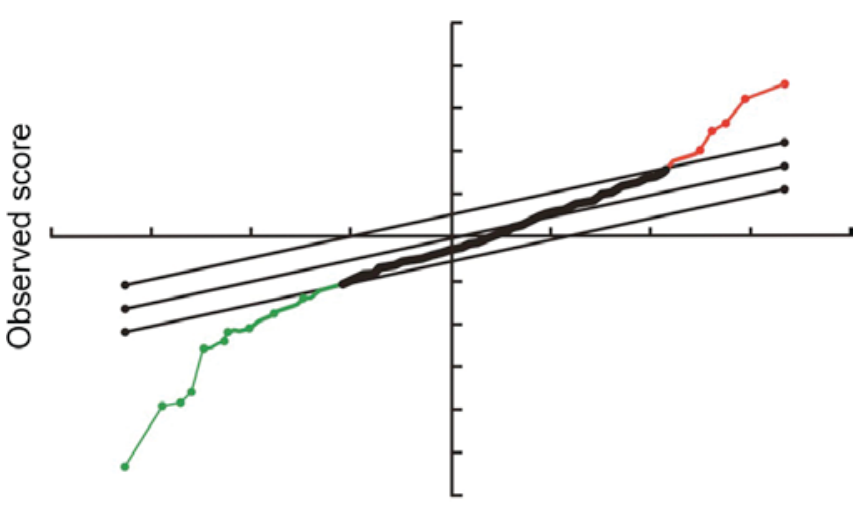

Expected score

Figure 2. microRNA genes identified in laryngeal cancer tissues using significance analysis of microarrays. The analysis demonstrated that 11 genes were highly expressed (red) and 114 genes had low expression levels (green) in laryngeal cancer tissues.

\section{Results}

RNA extraction and quality analysis. $\mathrm{A}_{260} / \mathrm{A}_{280}$ values for all RNA specimens were between 1.9 and 2.1, as determined by the spectrophotometer. Agarose gel electrophoresis exhibited sharp bands for the 28s and 18s RNA, with the intensity of 28 s being twice as strong as 18 s (data not shown). The results demonstrated that the extracted RNA was of high quality, allowing for subsequent analysis to be conducted.

MiRNA microarray. A total of 2,662 differentially expressed miRNAs were screened by miRNA microarray. Following the removal of non-human miRNA and miRNA that could not be detected, 780 miRNA were compared and analyzed with SAM software (FDR, 0.05). The results demonstrated that there were 11 and 114 miRNA with significantly upregulated and downregulated expression in laryngeal carcinoma 


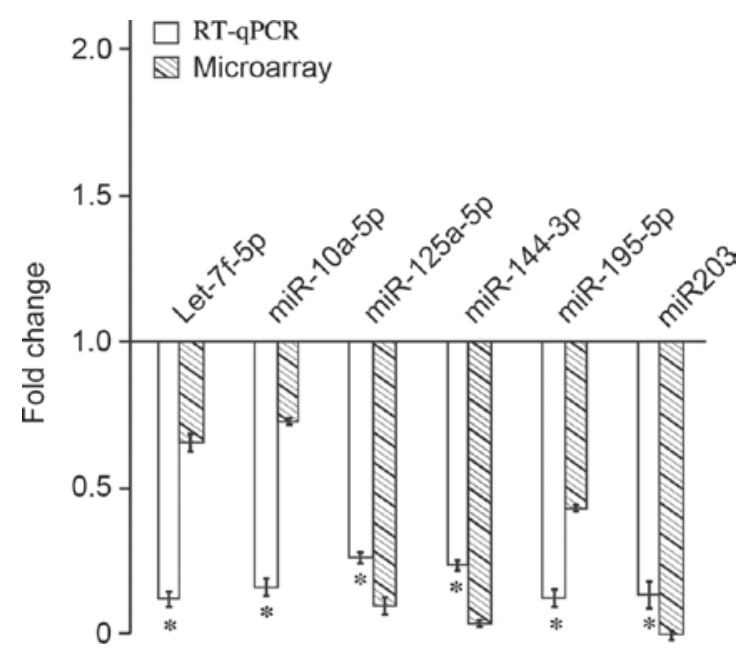

Figure 3. Comparison of the fold change results obtained from RT-qPCR and microarray analysis data for selected miRNAs. Both miRNA microarray and RT-qPCR demonstrated that the expression of miRNA let-7f-5p, miR-10a-5p, miR-125a-5p, miR-144-3p, miR-195-5p and miR-203 were downregulated in laryngeal cancer tissue compared with normal laryngeal tissue. RT-qPCR, reverse transcription-quantitative polymerase chain reaction; miRNA, microRNA. ${ }^{*} \mathrm{P}<0.05$ vs. normal laryngeal tissue (baseline).

tissues, respectively, when compared with normal tissues (Figs. 1 and 2).

$R T-q P C R$ verification. Expression levels of miRNA let-7f-5p miR-10a-5p, miR-125a-5p, miR-144-3p, miR-195-5p and miR-203 were significantly different between 32 biopsies of laryngeal carcinoma and their corresponding adjacent normal laryngeal mucosa $(\mathrm{P}<0.05)$. The miRNA quantitative $\mathrm{PCR}$ dissolution curves harbored single peaks, indicating excellent PCR amplification specificity. Compared with the RT-qPCR results, the microarray results demonstrated that the expression levels of the six miRNA were all downregulated. These results were consistent with the results obtained from the microarray analysis (Fig. 3).

MTT assay. Hep2 laryngeal carcinoma cells were transfected with a miR-125a-mimic, miR-125a-inhibitor and a negative control. The results demonstrated that the number of live cells transfected with miR-125a-mimics was significantly decreased compared with the negative control group (day 1 , $\mathrm{P}<0.05$; days 3, 5 and $7, \mathrm{P}<0.01$; Fig. 4), indicating inhibited viability. In contrast, the number of live cells transfected with miR-125a-inhibitor was significantly increased and the viability was considerably enhanced (day $1, \mathrm{P}<0.05$; days 3,5 and 7, P<0.01; Fig. 4).

Clone formation. Results of the cell clone formation experiment demonstrated that clone formation of Hep2 cells transfected with miR-125a-mimics $(7.6 \pm 0.9 \%)$ was significantly lower than the normal control group $(17.5 \pm 0.9 \%$; $\mathrm{P}<0.01$; Fig. 5). In contrast, clone formation of Hep2 cells transfected with miR-125a-inhibitor $(21.4 \pm 0.8 \%)$ was significantly higher than the normal control group $(10.0 \pm 0.7 \%$; $\mathrm{P}<0.01$; Fig. 5). These results demonstrate that the expression of miR-125a-5p inhibits the proliferation of Hep2 laryngeal cancer cells.

\section{Discussion}

In the present study, miRCURY LNA v.16.0 microarray was utilized to analyze a total of 42 pairs of frozen laryngeal carcinoma tissues and their adjacent normal tissues to obtain the complete expression profile of miRNA. Using SAM software, differences in the expression levels of miRNA were observed between the laryngeal carcinoma and normal tissues. Further analysis, by RT-qPCR, of 32 pairs of the samples demonstrated that the miRNA let-7f-5p, miR-10a, miR-125a-5p, miR-144-3p, miR-195-5p and miR-203 were all significantly downregulated in laryngeal carcinoma tissue compared with normal laryngeal tissue. The results were consistent between microarray and RT-qPCR, suggesting that the results obtained from microarray analysis were reliable. MTT and colony formation assays demonstrated that miR-125a-5p inhibited the viability and proliferation of Hep2 laryngeal carcinoma cells; therefore, miR-125a-5p may be a novel target for laryngeal carcinoma biotherapy.

Microarray technology is an important means of studying miRNA expression profiles. There are $\sim 2,000$ types of identified miRNA, thus a high-efficiency, high-throughput detection technology is required to analyze such large numbers of miRNA. Compared to the traditional methods of RNA analysis, miRNA microarray technology presents several obvious advantages: Firstly, high-throughput can simultaneously detect thousands of miRNA; secondly, the technology has high sensitivity and efficiency; and, thirdly, $1 \mu \mathrm{g}$ total RNA is sufficient for all tests. The present study utilized highly sensitive and specific microarray, which contains 1,890 miRNA probes to detect the microarray chip of all miRCURY LNA v.16.0 miRNA in the miRbase database to ensure the reliability and accuracy of the results as far as possible.

RT-qPCR is widely regarded as the gold standard for nucleic acid detection and quantification (16). Due to the presence of false positive results associated with the miRNA microarray utilized in the present study, the differential miRNA expression levels obtained required further validation; therefore, RT-qPCR is often used to verify specific miRNA found in microarray gene chips or other high-throughput experiments. Chen et al (17) designed stem-loop RT-qPCR with a simple, highly sensitive and highly specific characteristic, which has become the recommended method of quantitative detection of miRNA. The $2^{-\Delta \Delta C q}$ method was used to quantify the expression level of miRNA in the present study (15). The results of RT-qPCR and microarray assay were consistent and significant in exhibiting the downregulation of miR-125a-5p in laryngeal carcinoma tissues, and miR-125a-5p was selected for further investigation using MTT and clone formation assay.

The present miRNA profiling study had a number of limitations. For example, the majority of samples were head and neck squamous cell carcinomas, and few were laryngeal cancer samples. Although the locations of head and neck tumors are closely related, the throat, as a part of the respiratory system, is different to the mouth, pharynx and other components of the digestive system $(18,19)$. Laryngeal cancer is distinct from oral cancer, oropharyngeal cancer and hypopharyngeal carcinoma of head and neck squamous cell carcinoma in terms of key clinical pathological and prognostic features of the patients, such as the incidence ratio 

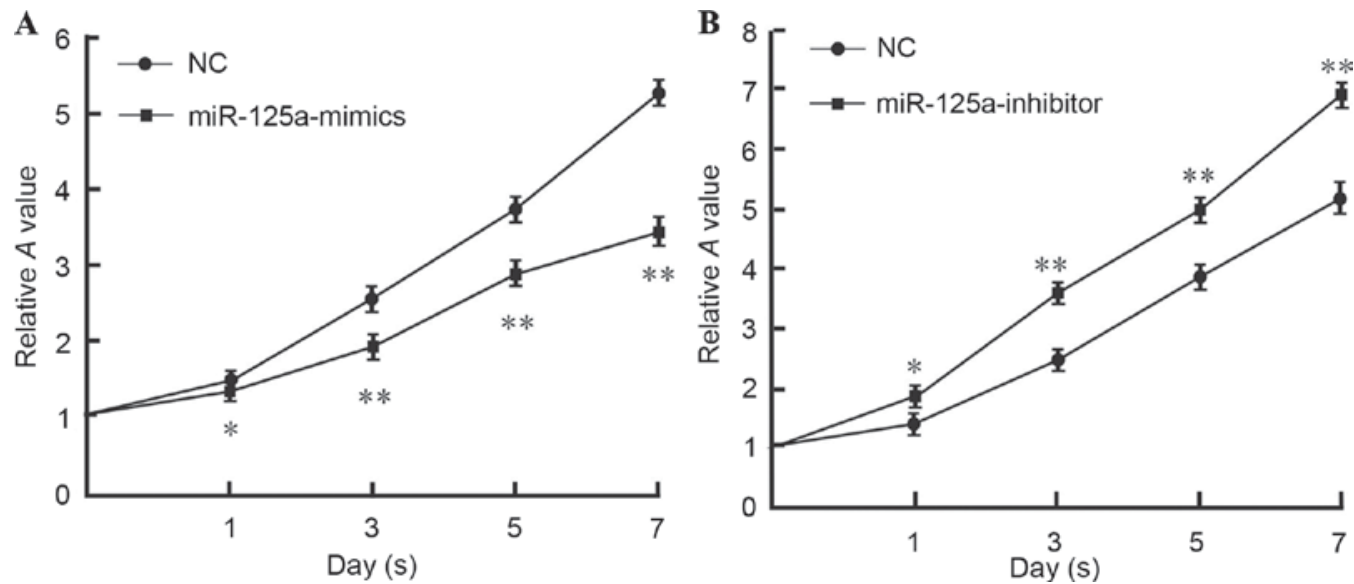

Figure 4. Results of methylthiazolyldiphenyl-tetrazolium bromide assay. (A) Upregulation of miR-125a using miR-125a-mimics suppresses cell growth of Hep2 cells compared with the NC group. (B) Inhibition if miR-125a using miR-125a-inhibitors promotes cell growth of Hep2 cells. Data are presented as mean \pm standard deviation $(\mathrm{n}=3)$. " $\mathrm{P}<0.05$ and ${ }^{* *} \mathrm{P}<0.01$ vs. NC. miR, microRNA; Hep2, human epithelial type 2; NC, normal control.

$\mathbf{A}$

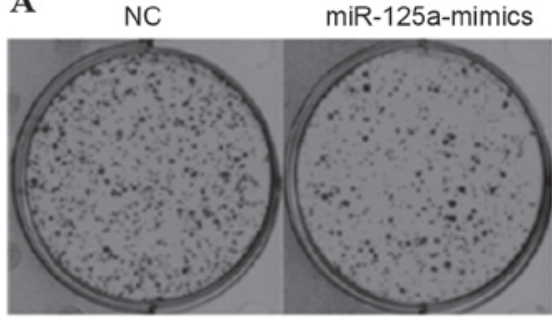

B

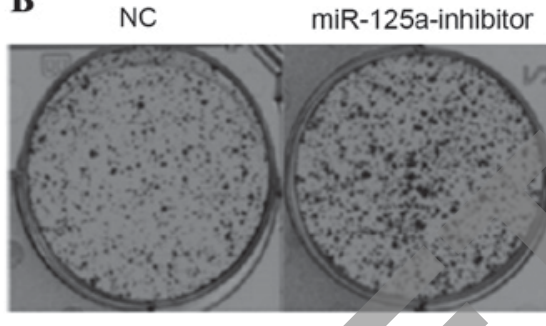

Figure 5. Colony-forming assay (crystal violet staining). Hep2 laryngeal carcinoma cells were transiently transfected with miR-125-mimics, miR-125-inhibitors or a scrambled sequence oligonucleotide as a NC (A) Hep2 laryngeal carcinoma cells transfected with miR-125-mimics exhibited a significant decrease in colony number compared with the NC. (B) Hep2 laryngeal carcinoma cells transfected with miR-125-inhibitor exhibited a significant increase in cell colony numbers compared with the NC. Data are presented as mean \pm standard deviation $(\mathrm{n}=3){ }^{* * *} \mathrm{P}<0.01$ vs. NC. Hep2, human epithelial type 2; miR, microRNA; NC, normal control.

of laryngeal carcinoma being higher in males than in other head and neck cancers (19). Furthermore, gene hybridization experiments have demonstrated that there is a significant difference in chromosome type when comparing laryngeal and other head and neck squamous cell carcinomas (20); therefore, the non-discriminatory study of all head and neck squamous cell carcinoma may have resulted in an unwanted bias and affected the validity of the results of the present experiment. In addition, the present study utilized a small specimen number. When considering differences between studies, some studies have used paraffin to embed tissues, and various studies have conducted gene chip tests without the RT-qPCR validation, which may have generated confounded and unreliable results $(21,22)$.
In the present study, results were consistent with head and neck squamous cell carcinoma expression of the miRNA miR-10a-5p (23), miR-125a-5p (14), miR-203 (24) and miR-144-3p (25). Gene chip detection was used to identify the expression levels of miR-125a-5p, miR-203 and miR-144-3p in previous studies $(14,24,25)$. The present study utilized RT-qPCR to validate miRNA expression and a preliminary investigation of the involvement of miR-125a-5p in the proliferation of Hep2 laryngeal cancer of cells was conducted.

The present study identified miRNA, including let-7f-5p and miR-195-5p, that have no previous or previous controversial reports $(26,27)$. Let-7, including let-7a, b, $\mathrm{c}, \mathrm{d}$, e and $\mathrm{f}$, was one of the earliest discovered miRNA in Caenorhabditis elegans (28). Some reports $(14,23)$ have demonstrated that let-7i exhibited high expression in head and neck squamous cell carcinoma, whereas let-7a, c and e exhibited low expression. In the present study, the expression of let-7f-5p was low in laryngeal carcinoma tissues and, therefore, contradicted the results reported in a study by Tran et al (29). This may be explained by the fact that Tran et al (29) conducted gene chip detection without subsequent verification for let-7f in tongue, amygdala, pharynx, larynx and other head and neck cancer. The present study was conducted on fresh frozen laryngeal cancer tissue with independent verification by RT-qPCR, which further proved that the single disease (laryngeal cancer) was advantageous, in terms of prognosis, when compared with multiple different head and neck tumors. Gene hybridization experiments indicate that the chromosome type of laryngeal carcinoma differed from other head and neck squamous cell carcinomas; therefore, laryngeal, oropharyngeal and hypopharyngeal carcinoma have differences in terms of key clinical pathological features and prognosis of the patients (19). This highlights that the study of a single disease, such as laryngeal cancer, is advantageous over a combination study of various types of head and neck tumors in multiple sites. miR-195 is an important member of the miRNA-15/16/195/424/497 family that is downregulated in various types of cancer, including liver, gastric, breast, bladder cancer and chronic lymphocytic leukemia, which suggests that miR-195 may be an important tumor suppressor (30). The present study represents the first report to demonstrate that miR-195 has reduced expression in 
laryngeal cancer; however, further investigation is required to confirm this result at the cellular level.

In the present study, the expression of miR-125a-5p was downregulated in laryngeal carcinoma tissue compared with normal tissue, as identified by gene chip analysis and RT-qPCR. The effect of miR-125a-5p on the proliferation of Hep2 laryngeal cancer cells was also investigated at the cellular level. The results demonstrated that transfection with miR-125a-mimics inhibits cell proliferation and transfection with miR-125a-inhibitor increases cell proliferation. Zhang et al (31) demonstrated that miR-125 and other miRNA present in norcantharidin promoted the apoptotic process of chronic myeloid leukemia K562 cells by regulating oncogenes and anti-oncogenes (including Bcl-2 and p53). It has been demonstrated that miR-125a expression is downregulated in liver cancer tissues and cell lines and was related to the pathological features of cancer cell invasion (32). Upregulated expression of miR-125a may inhibit matrix metalloproteinase 11 and vascular endothelial growth factor, inhibiting the malignant phenotype of hepatocellular carcinoma cells, suggesting that miR-125 may have profound therapeutic potential in the treatment of tumors and prognostic markers.

\section{References}

1. Lu ZM: Differential microRNA expression profiling in laryngeal cancer and study of mir-125a on the proliferation of laryngeal cancer cell line. Southern Med Univ, Master's Degree, 2013

2. Jaseviciene L, Gurevicius R, Obelenis V, Cicenas S and Juozulynas A: Trends in laryngeal cancer incidence in ithuania: A future perspective. Int J Occup Med Environ Health 17: 473-477, 2004.

3. Lu ST, Wei KR, Yu BH, et al: Incidence trend analysis of laryngeal carcinoma. Xian Dai Zhong Liu Yi Xue 12: 158-160, 2004 (In Chinese)

4. Hoffman HT, Porter K, Karnell LH, Cooper JS, Weber RS, Langer CJ, Ang KK, Gay G, Stewart A and Robinson RA: Laryngeal cancer in the United States: Changes in demographics, patterns of care and survival. Laryngoscope 116 (Suppl 111): S1-S13, 2006.

5. Martin DA, James O, Allen SL and John EN (eds.): Clinical oncology. Peking: Scientific Publishing Agency 1801, 2001.

6. Hashibe M, Boffetta P, Zaridze D, Shangina O, Szeszenia-Dabrowska N, Mates D, Fabiánová E, Rudnai P and Brennan P: Contribution of tobacco and alcohol to the high rates of squamous cell carcinoma of the supraglottis and glottis in central Europe. Am J Epidemiol 165: 814-820, 2007.

7. Potter CS, Wang Z, Silva KA, Kennedy VE, Stearns TM, Burzenski L, Shultz LD, Hogenesch H and Sundberg JP: Chronic proliferative dermatitis in sharpin null mice: Development of an autoinflammatory disease in the absence of B and Tlymphocytes and IL4/IL13 signaling. PLoS One 9: e85666, 2014.

8. Li P and Wang X: Relationship between Myc gene family and laryngeal cancer. Shi Yong Zhong Liu Xue Za Zhi 16: 67-69, 2002 (In Chinese).

9. Pietruszewska W, Durko M and Kobos J: Alterations of cell cycle regulating proteins: $\mathrm{Rb}, \mathrm{p} 21$ and p16 in laryngeal cancer. Otolaryngol Pol 61: 951-957, 2007 (In Polish).

10. Croce CM and Calin GA: MiRNAs, cancer and stem cell division. Cell 122: 6-7, 2005.

11. Ambros V: The functions of animal microRNAs. Nature 431: 350-355, 2004

12. Esquela-Kerscher A and Slack FJ: Oncomirs: MicroRNAs with a role in cancer. Nat Rev Cancer 6: 259-269, 2006.
13. Wang Z, Potter CS, Sundberg JP and Hogenesch H: SHARPIN is a key regulator of immune and inflammatory responses. J Cell Mol Med 16: 2271-2279, 2012.

14. Ramdas L, Giri U, Ashorn CL, Coombes KR, El-Naggar A, Ang KK and Story MD: MiRNA expression profiles in head and neck squamous cell carcinoma and adjacent normal tissue. Head Neck 31: 642-654, 2009.

15. Livak KJ and Schmittgen TD: Analysis of relative gene expression data using real-time quantitative PCR and the 2(-Delta Delta C(T)) method. Methods 25: 402-408, 2001.

16. Schmittgen TD, Lee EJ, Jiang J, Sarkar A, Yang L, Elton TS and Chen C: Real-time PCR quantification of precursor and mature microRNA. Methods 44: 31-38, 2008.

17. Chen C, Ridzon DA, Broomer AJ, Zhou Z, Lee DH, Nguyen JT, Barbisin M, Xu NL, Mahuvakar VR, Andersen MR, et al: Real-time quantification of microRNAs by stem-loop RT-PCR. Nucleic Acids Res 33: e179, 2005.

18. Zhang C, Zeng X, Li Z, Wang Z and Li S: Immunoglobulin A nephropathy: Current progress and future directions. Transl Res 166: 134-144, 2015

19. Jemal A, Siegel R, Ward E, Murray T, Xu J, Smigal C and Thun MJ: Cancer statistics, 2006. CA Cancer J Clin 56: 106-130, 2006.

20. Huang Q, Yu GP, Mccormick SA, Mo J, Datta B, Mahimkar M, Lazarus P, Schäffer AA, Desper R and Schantz SP: Genetic differences detected by comparative genomic hybridization in head and neck squamous cell carcinomas from different tumor sites: Construction of oncogenetic trees for tumor progression. Genes Chromosomes Cancer 34: 224-233, 2002.

21. Li YD, Gowhere Ali and Hong SL: Relationship between single nucleotide polymorphism in genome carcinoma tissue and tumor metastasis. Aca J Sec Mil Med Univ 32: 713-716, 2011.

22. Wang J: The application of gene chip technology on head and neck neoplasm. Otol Forei Med Sci 29: 382-385, 2005.

3. Hui AB, Lenarduzzi M, Krushel T, Waldron L, Pintilie M, Shi W, Perez-Ordonez B, Jurisica I, O'Sullivan B, Waldron J, et al: Comprehensive microRNA profiling for head and neck squamous cell carcinomas. Clin Cancer Res 16: 1129-1139, 2010.

24. Zhong Q, Fang JG, Huang ZG, et al: A preliminary study of miRNA expression in laryngeal squamous cell carcinoma. Chin J Cancer Prev Control 17: 1073-1076, 2010 (In Chinese).

25. Wang P, Fu T, Wang XR, et al: Preliminary study on the differential expression of miRNA and normal mucosa in laryngeal squamous cell carcinoma by microarray analysis. Lin Chuang Er Bi Yan Hou Tou Jing Wai Ke Za Zhi 24: 535-538, 2010 (In Chinese).

26. Wu YB, Shen ZS, Yu X, et al: Research advances of microRNAs related to laryngeal carcinoma. Ji Chu Yi Xue Yu Lin Chuang 32: 583-586, 2012 (In Chinese).

27. Yang J,Zhang Q, Dong JQ, Chang XH and He XJ: Overexpression of high mobility group A2 and its correlation with microRNA let-7 falimy in serous ovarian cancers. Beijing Da Xue Xue Bao 44: 749-754, 2012 (In Chinese).

28. Chen FY and Chen QY: Research progress in the correlation between MicroRNA let-7 and cancer. Yi Xue Zong Shu 17: 1797-1800, 2011 (In Chinese).

29. Tran N, Mclean T, Zhang X, Zhao CJ, Thomson JM, O'Brien C and Rose B: MicroRNA expression profiles in head and neck cancer cell lines. Biochem Biophys Res Commun 358: 12-17, 2007.

30. He JF, Luo YM, Wan XH and Jiang D: Biogenesis of miRNA-195 and its role in biogenesis, the cell cycle and apoptosis. J Biochem Mol Toxicol 25: 404-408, 2011.

31. Zhang S, Li YJ, Zhang C, et al: Study on the role of K562 in apoptosis induced by norcantharidin in miRNA. Zhong Guo Bing Li Sheng Li Za Zhi 27: 499-503, 2011 (In Chinese).

32. Bi Q, Tang S, Xia L, Du R, Fan R, Gao L, Jin J, Liang S, Chen Z, $\mathrm{Xu} \mathrm{G}$, et al: Ectopic expression of miR-125a inhibits the proliferation and metastasis of hepatocellular carcinoma by targeting MMP11 and VEGF. PLoS One 7: e40169, 2012. 